\title{
Intravertebral insertion of Interbody fusion cage via transpedicular approach for the treatment of stage III Kümmell disease区a technical note and case presentation
}

\author{
changjun Chen
}

Shandong University Qilu Hospital

Xianlei Gao

Shandong University Qilu Hospital

Hao Li

Shandong University Qilu Hospital

Xin Pan

Shandong University Qilu Hospital

Songgang Wang ( $\square$ wasoga@sina.cn )

Shandong University Qilu Hospital https://orcid.org/0000-0002-7086-4774

\section{Technical note}

Keywords: Modified surgery, interbody cage, new application, advanced Kümmell disease, spine stabilization

Posted Date: July 17th, 2020

DOI: https://doi.org/10.21203/rs.3.rs-31303/v2

License: (c) (i) This work is licensed under a Creative Commons Attribution 4.0 International License. Read Full License 


\section{Abstract}

Background Kümmell disease usually occurs in the elderly osteoporosis population and develops gradually into symptomatic, progressive kyphosis of the spine. Several alternative surgical procedures, including vertebroplasty, kyphoplasty, and osteotomy, can be chose for the treatment of the disease. However, current surgical methods to deal with stage III Kümmell disease are less satisfying. Method A modified technique of intravertebral insertion of interbody fusion cage with posterior spine stabilization was applied to treat stage III Kümmell disease. Results This study details a modified technique applied in a patient with stage III Kümmell disease, showing significant improvement in pain relief, anterior column height recovery, and kyphotic angle correction. And no complications was reported during our follow-up. Conclusions Intravertebral insertion of interbody fusion cage via transpedicular approach provides advantages of acceptable correction of kyphosis, bony fusion, minimal invasion. Thus, our method was a good alternative choice for stage III Kümmell disease.

\section{Background}

Kümmell disease, also known as avascular necrosis after vertebral compression fracture (VCF), is increasingly threatening the health of elderly population, and it is characterized by a particular sign called "intravertebral vacuum". This vacuum sign usually occurs after osteoporotic VCFs, therefore, osteoporosis is always viewed as the basic cause of this disease (1). Osteoporosis, avascular necrosis of the vertebral body, and biomechanical changes following fracture are the main factors that involve of the formation and progression of this disease. Usually, the interruption of blood supply due to small tiny fractures and the insufficiency of neovascularization are likely to induce the avascular necrosis of the vertebral body; Then, fractured vertebra with a compression state results in the loss of vertebral height and kyphosis, which further changes of the biomechanics environment of vertebra and might lead to more severe fracture (2). Therefore, under this negative biomechanical balance, fracture union and stabilization are difficult and vertebral pseudarthrosis can be easily formed.

Based on the severity of the disease, it could be managed conservatively or surgically. Conservative treatment include bed rest, traction, wearing a brace, analgesics and anti-osteoporosis drugs; However, surgical interventions are recommended if the pain worsen, becomes persistent or new symptoms like radiculopathy, increasing neurological deficit, or significant deformity occurs (3). The current surgical options for management of Kümmell disease are vary, including percutaneous vertebroplasty (PVP) and percutaneous kyphoplasty (PKP) which are proved to be effective methods for the management of Kümmell's disease without neurological symptoms, and can relieve the pain and restore the height of vertebra to some extent (4). With advanced Kümmell's disease, especially stage III, PVP or PKP are less safe and effective to restore the height of vertebra and provide stability of the spine, therefore titanium mesh bone grafting or osteotomy are the alternative measures for stage III Kümmell's disease(5-8). However, these procedures are technically demanding and highly risky, and has the characteristics of high blood loss, long duration of surgery and increases the rate of long-term complications, such as loss of 
correction rate, subsequent vertebral fractures(9). Therefore, less invasive and more safe methods are still needed for the treatment of Kümmell disease, especially the advanced stages.

Management of Kümmell's disease, especially stage III Kümmell disease is still controversial, and no standard surgical options or single effective treatments are available so far. More importantly, the method of titanium mesh bone grafting or osteotomy surgery for the treatment of stage III Kümmell disease is not a safe procedure and high risk of complications should be anticipated. In this setting, it is essential to focus on the primary pathophysiologic process and reduce surgical-associated complications. Therefore, we reported the surgical details and satisfactory outcomes of an old adult male diagnosed with Kümmell disease stage III, who was treated by less invasive method of transpedicular interbody cage insertion with posterior spine stabilization.

\section{Methods}

\section{Patient presentation}

The Institutional review board (IRB) of the author's affiliated institution approved this treatment, and informed consent has been obtained from the patient. A 61-year-old male with a history of Parkinson's disease and brain atrophy was presented to our department with a severe pain in the lumbosacral region after a fall one week ago. Three months prior to the admission, the patient fell down and had a pain in the lumbosacral region. The pain was aggravated during activity and was unbearable after another accidental fall one week ago. Physical examination showed that the patient has kyphosis deformity in the standing position. There was compression pain in spinous process and para-vertebral region of T12 horizontal without lower extremity radiation pain. Visual analog scale (VAS) of low-back pain was 7 points. Pre-operative X-rays showed T12 compression fracture and kyphosis (Figure 1囚A/B). CT scans (Figure1, C) showed T12 vertebral compression fracture with an intra-vertebral vacuum and kyphosis. The MRI study showed that the posterior wall of the fractured vertebral body was damaged and retropulsed bone fragment invaded the spinal canal with mild cord compression (Figure1, D). The patient was diagnosed with stage III Kümmell disease

Due to the complexity of the patient's symptoms, it was proposed to carry out a novel surgical approach: intravertebral insertion of interbody fusion cage via transpedicular approach combined with posterior spine stabilization

\section{Surgical procedure}

After the general anesthesia, the patient was placed in a prone position and a pair of soft cushion was placed under his chest and hip, making the abdomen suspended to maintain the over-extension of the spine, which can contribute to reduction of the fractured vertebral body and decompression of spinal canal via physical distraction (Figure 2, A). The median longitudinal incision was made to expose the T9L3 spinous processes, laminas and zygapophysial joints. Then, pedicle screws were inserted into the 
targeted vertebrae T9, T10, T11, L1, L2, and L3 through bilateral pedicles under C-arm guidance (Figure 2, $\mathrm{B} ; \mathrm{C})$. The fixation segment was determined based on the severity of osteoporosis during the operation.

Based on preoperative CT scan, we operated on the severe side of the vertebrae, osteotomy of the transverse process was made and the vertebral pedicle was exposed.

Then we reamed and enlarged the pedicle tunnel with a hand bone drill or osteotome with the help of a guide pin when necessary. Because the superior and lateral cortex of the pedicle can be violated without dangers of neurological sequelae. Thus, in order to establish a large enough safe zone, we removed the bone in the lateral cortex even the outer region of the lateral cortex; If necessary, the inferior cortex of the pedicle could also be removed. However, when dealing with the inferior cortex of the pedicle, care must be taken to protect the nerve root. The working channel is preliminary established.

We pre-determined the cage height according to the height of the vertebral body in overextension position on an intraoperative X-ray fluoroscopy. And we used carbon fiber-reinforced polymer (CFRP) fusion cages (23 or $27 \mathrm{~mm}$ long and 7-17mm high; Vertebral Body Spacer, Johnson \& Johnson, USA). The cage used in our case was $23 \mathrm{~mm}$ long and $12 \mathrm{~mm}$ high. We tried sequentially larger cages to maximize the height of collapsed vertebra. In details, the $7 \mathrm{~mm}$ test mold was gently wedged into the gap along the pedicle tunnel in order to gradually re-open the collapsed vertebral body; Then, $8 \mathrm{~mm}$ to $17 \mathrm{~mm}$ test molds were inserted into the vertebral body respectively until the height of the vertebral body was satisfyingly restored under carm fluoroscopy (Details were shown in Figure 2, D). Then, we chose the appropriate cage size and fill enough autologous bone graft into it. The lesions and soft tissues within the vertebral body were completely cleaned up until the healthy bone, and adequate amount of bone grafts compactly filled up the vacuum space. Accordingly, the cage was inserted into the vertebral body through the pedicle tunnel under $\mathrm{C}$-arm fluoroscopy. We made sure that the cage position was good and the vertebral height was restored satisfactorily. At last, adequate amount of bone grafts were used to fill up the space of the pedicle tunnel. Then, pedicle screws were fixed and fused after reconstruction of the spine curve using a bendable titanium rod. The intraoperative blood loss was about $200 \mathrm{~mL}$ and the operation time was 120 minutes.

The intraoperative $\mathrm{x}$ rays showed that the height of vertebra was restored and kyphosis was totally corrected immediately after operation; The height of the intervertebral discs were also recovered to some extent (Figure 2, E). The patient was treated with antibiotics for three days postoperatively and antiosteoporosis drugs were prescribed after the operation. The drainage tube was removed two days postoperatively when the drainage volume was less than $50 \mathrm{~mL} / 24 \mathrm{~h}$. Patient was allowed to stand or walk with a custom-made plastic orthosis at three days after operation. The plastic orthosis was kept for at least 3 months and adequate physical exercises were recommended.

\section{Results}

\section{Evaluation and follow-up}


After treatment, the patient's pain and kyphosis were significantly reduced. Follow-up CT scans on day three postoperatively showed adequate stabilization, successful kyphosis correction and sufficient bone grafting (Figure 3, A/B). Radiographs after one week postoperatively also showed adequate stabilization and kyphosis correction (Figure 3,C/D). Radiological investigations at 12 weeks follow-up were available and showed good bone formation and union (Figure 3, E/F). However, he was died because of an accidental fall within 6 months. And he remained in good condition before his death and no postoperative complications were reported during our follow-up.

\section{Discussion}

Kümmell disease is a specific condition of vertebral compression fractures $\Downarrow$ which is a typically manifestation of failed fracture healing process, and conservative treatments are usually ineffective. Although the optimal therapeutic methods remain controversial, surgical interventions are still needed to control the refractory pain or kyphosis. Though the optimal therapeutic methods remain controversial, surgical interventions are still needed to control the refractory pain or kyphosis. Various surgical procedures have been proposed for the management of Kümmell disease, such as PVP or PKP, which have satisfying effect to relieve clinical symptoms and improve functional status $(10,11)$. However, stage III Kümmell disease is more challenging for orthopaedic surgeons, because these patients have broken posterior cortex and obvious intravertebral instability. Conventional PVP or PKP could neither reconstruct the intravertebral stability nor contribute to the union of fracture, and the risks of cement leakages during the operation or the needs of revision surgery to remove the dislocated polymethylmethacrylate (PMMA) cement might lead to much more damages to the elderly patients $(7,12)$.Moreover, due to the loading shifts of adjacent vertebra, subsequent risks of vertebral fracture are high $₫$ And impaired nutrient supply to the disc due to the heat released by PMMA might induce degenerative changes in the adjacent disc, complicating the existing situation $(13,14)$.

For advanced Kummell disease, most surgeons choose open surgery to decompress spinal cord and stabilize the spine, however, current surgical options are complicated and risky for these patients. Both of anterior reconstruction (AR) and posterior osteotomy (PO) are common treatment methods for the situation mentioned above, of which $A R$ approach could resect the bony fragments and provide anterior column support and PO approach could dissect the cortex by posterior spinal shortening osteotomy and correct kyphotic deformity. However, there are many disadvantages, such as AR approach might detach the diaphragm or open either the thoracic cavity or the retroperitoneal space, which could compromise lung function or injure the internal organs; longer operation time; cause of kyphosis due to vertebral body or graft re-collapse(9). In view of patients' advanced age, serious comorbidities and severe osteoporosis, AR approach is inappropriate for these individuals because of its high risks and invasiveness. In contrast, most surgeons are more familiar with the posterior approach. PO and fixation can provide relatively secure fixation with less complications, and stable fixation could help early mobilization with lower incidence of implant-related complications, such as loose screw, screw fracture and screw disconnection (15). However, Osteotomy is a challenging and complicated procedure that requires delicacy and 
surgeons with more experiences (6). Any error around the spinal cord or medullary cone would be dangerous and might lead to nerve impingement or dural tear (16).

A successful surgical procedure for treating advanced kummell disease in elderly patients must consider the biological and biomechanical factors of this condition. Autologous or allograft bone should be implanted after thoroughly removal of the necrotic bone tissues, so that to promote osteogenesis and angiogenesis. On the other hand, better mechanical support of the collapsed vertebral body and good fixation are required to maintain the biomechanical stability.

In this case, we reported a detailed approach of transpedicular interbody cage insertion with posterior spine stabilization to treat stage III Kümmell disease in elder patient. A similar approach by Mei L (8) et al were reported in the literature. They used a series of transpedicular body augments (a titanium spacer with bone-ingrowth porous surface) to treat Kümmell disease with cord compression (III stage), and successfully restored body height and corrected kyphosis. Although there are different sizes of the augments available, their method has not been used widely and commonly in clinical practice. The custom-made tools that were needed for their method were hard to obtain for others. Although the porous surface of the augments might contribute to bone ingrowth, the shape of the augments can't prevent itself from slip backward and it might not maintain stability in the implanted cancellous bones during the earlier time postoperatively. In addition, their method required making bilateral pedicle tunnels of the fractured vertebral body, which might influence the stability of the spine to some extent. Likewise, another similar method reported by Lee SH (17) et al used two kinds of different shaped polyetheretherketone (PEEK) interbody cages, which are CRESCENT cage and CAPSTONE cage. The CRESCENT cages are comparatively expensive, and its shape limits the function of wedged expansion, compared to bullethead. Also, it is difficult to control the direction when inserting them. While CAPSTONE cages have weak bulletshaped head and contain less amount of bone grafts, which might influence the insertion and bone fusion (Figure 6 B).

In this case, our modified approach with large enough space enables a sizeable cage to be inserted, and the carbon fiber-reinforced polyetheretherketone (CFRP) cages that we used here are widely in use clinically. This type of cage was initially designed for posterior lumbar interbody fusion (Figure 4A), where its wedged bullethead can facilitate cage insertion (Figure 4B), tine-teeth devise can help maintain stability, and tantalum beads enable conventional imaging methods for evaluation of bone fusion postoperatively (Figure 4A), allowing for better follow-up studies (18). In addition, CFRP fusion cages can accommodate much more graft material and larger contact surface than CAPSTONE cages (Figure 4C), which may improve fusion rates. Furthermore, CFRP implants have better biological and mechanical properties compared to titanium implants, which can transfer high stresses and forces efficiently due to their ability to sustain both tension and compression (19). And our approach is less invasive and the possible risks of injuring the nerve root could be reduced with surgeons of good skills and experience. Moreover, the reduction of the fractured vertebrae by this method is easier to achieve but must be with cautionary in patients with chronic conditions of fracturees or ossified posterior longitudinal ligament (20). Finally, this method has the least interference on blood circulation of intervertebral disc, the 
debridement of fracture sites and bone grafting might help the recovery of blood circulation of the disc, which prevent the degeneration of the disc[14]. The limitation of this method including the necessity to remove unilateral transverse process and part of the pedicle in order to insert a bigger cage to restore the height the compressed vertebra. However, this doesn't affect the spinal stability because of the existence of posterior pedicle screw. Furthermore, we found that there is no need to remove the superior or the inferior cortex of the pedicle after proficiency, because the fractured vertebral body can easily be expanded via the wedge-shaped cage.

\section{Conclusion}

Intravertebral insertion of interbody fusion cage via transpedicular approach provides advantages of acceptable correction of kyphosis, bony fusion, minimal invasion. Our modified method was less invasive and provides enough operative space for cage insertion and bone graft, which emerges as a good alternative choice for stage III Kümmell disease. However, more clinical and long-term follow-up studies are needed to investigate the effects of this method.

\section{Declarations}

\section{Ethics approval and consent to participate}

Our Institutional review board (IRB) at Shandong University Qilu Hospital was consulted prior to undertaking this case report, and written informed consents were obtained from the participant(In Chinese). All experiments were performed in accordance with relevant guidelines and regulations.

Consent for publication The authors have seen the manuscript and approved to submit to your journal.

Availability of data and material The datasets used and/or analyzed during the current study are available from the corresponding author on reasonable request.

Competing interests The authors declare that they have no conflict of interest.

Funding The authors state that this work has not received financial support.

Authors' contributions $\mathrm{CJ} C, \mathrm{XL}$ G and $\mathrm{H} L$ participated in the drafting, writing, and revising of the manuscript. X P and SG W participated in the conception and design of the study. Both of them contributed to analysis and interpretation of the data and they approved the final version of the manuscript to be submitted, and agreed to be accountable for all aspects of the work.

Acknowledgements The authors would like to thank the participating patient and the colleagues who made this work possible.

\section{References}


1. Matzaroglou C, Georgiou CS, Panagopoulos A, Assimakopoulos K, Wilke HJ, Habermann B, et al. Kummell's Disease: Clarifying the Mechanisms and Patients' Inclusion Criteria. The open orthopaedics journal. 2014;8:288-97.

2. He D, Yu W, Chen Z, Li L, Zhu K, Fan S. Pathogenesis of the intravertebral vacuum of Kummell's disease. Experimental and therapeutic medicine. 2016;12(2):879-82.

3. Li H, Liang CZ, Chen QX. Kummell's disease, an uncommon and complicated spinal disorder: a review. The Journal of international medical research. 2012;40(2):406-14.

4. Zhang J, Fan Y, He X, Meng Y, Huang Y, Jia S, et al. Is percutaneous kyphoplasty the better choice for minimally invasive treatment of neurologically intact osteoporotic Kummell's disease? A comparison of two minimally invasive procedures. International orthopaedics. 2018;42(6):1321-6.

5. Zhang X, Hu W, Yu J, Wang Z, Wang Y. An Effective Treatment Option for Kummell Disease With Neurological Deficits: Modified Transpedicular Subtraction and Disc Osteotomy Combined With Long-Segment Fixation. Spine. 2016;41(15):E923-30.

6. Mei L, Sang W, Chen Z, Lou C, Zheng L, Jin K, et al. Titanium mesh bone grafting combined with pedicle screw internal fixation for treatment of Ku[Combining Diaeresis]mmell disease with cord compression: A case report and literature review. Medicine. 2018;97(36):e12183.

7. Zhang C, Wang G, Liu X, Li Y, Sun J. Failed percutaneous kyphoplasty in treatment of stage 3 Kummell disease: A case report and literature review. Medicine. 2017;96(47):e8895.

8. Li KC, Li AF, Hsieh CH, Liao TH, Chen CH. Another option to treat Kummell's disease with cord compression. European spine journal : official publication of the European Spine Society, the European Spinal Deformity Society, and the European Section of the Cervical Spine Research Society. 2007;16(9):1479-87.

9. Liu F, Chen Z, Lou C, Yu W, Zheng L, He D, et al. Anterior reconstruction versus posterior osteotomy in treating Kummell's disease with neurological deficits: A systematic review. Acta orthopaedica et traumatologica turcica. 2018;52(4):283-8.

10. Chen GD, Lu Q, Wang GL, Zou J, Yang HL, Yang Y, et al. Percutaneous Kyphoplasty for Kummell Disease with Severe Spinal Canal Stenosis. Pain physician. 2015;18(6):E1021-8.

11. Zhang GQ, Gao YZ, Chen SL, Ding S, Gao K, Wang HQ. Comparison of percutaneous vertebroplasty and percutaneous kyphoplasty for the management of Kummell's disease: A retrospective study. Indian journal of orthopaedics. 2015;49(6):577-82.

12. Kim JE, Choi SS, Lee MK, Lee DK, Cho SI. Failed Percutaneous Vertebroplasty Due to Insufficient Correction of Intravertebral Instability in Kummell's Disease: A Case Report. Pain practice : the official journal of World Institute of Pain. 2017;17(8):1109-14.

13. Han S, Jang IT. Analysis of Adjacent Fractures after Two-Level Percutaneous Vertebroplasty: Is the Intervening Vertebral Body Prone to Re-fracture? Asian spine journal. 2018;12(3):524-32.

14. Feng Z, Chen L, Hu X, Yang G, Chen Z, Wang Y. Vertebral Augmentation can Induce Early Signs of Degeneration in the Adjacent Intervertebral Disc: Evidence from a Rabbit Model. Spine. 2018;43(20):E1195-e203. 
15. Cho Y. Corpectomy and circumferential fusion for advanced thoracolumbar Kummell's disease. Musculoskeletal surgery. 2017;101(3):269-74.

16. Hu Z, Ma C, Rong X, Zou S, Liu X. Immunomodulatory ECM-like Microspheres for Accelerated Bone Regeneration in Diabetes Mellitus. ACS applied materials \& interfaces. 2018;10(3):2377-90.

17. Lee SH, Jain V, Lee SH, Baek OK, Moon KH. Transpedicular Intrabody Cage Insertion with Posterior Spine Stabilization in Kummell Disease: Report of 2 Cases. World neurosurgery. 2018;116:236-40.

18. Brantigan JW, Neidre A. Achievement of normal sagittal plane alignment using a wedged carbon fiber reinforced polymer fusion cage in treatment of spondylolisthesis. The spine journal : official journal of the North American Spine Society. 2003;3(3):186-96.

19. Kromoser B, Gericke O, Hammerl M, Sobek W. Second-Generation Implants for Load Introduction into Thin-Walled CFRP-Reinforced UHPC Beams: Implant Optimisation and Investigations of Production Technologies. Materials (Basel, Switzerland). 2019;12(23).

20. Hur W, Lee JJ, Kim J, Kang SW, Lee IO, Lee MK, et al. Spontaneous air reduction of vertebra plana with Kummell's disease during vertebroplasty: subsequent experience with an intentional trial. Pain medicine (Malden, Mass). 2014;15(7):1240-2.

\section{Figures}

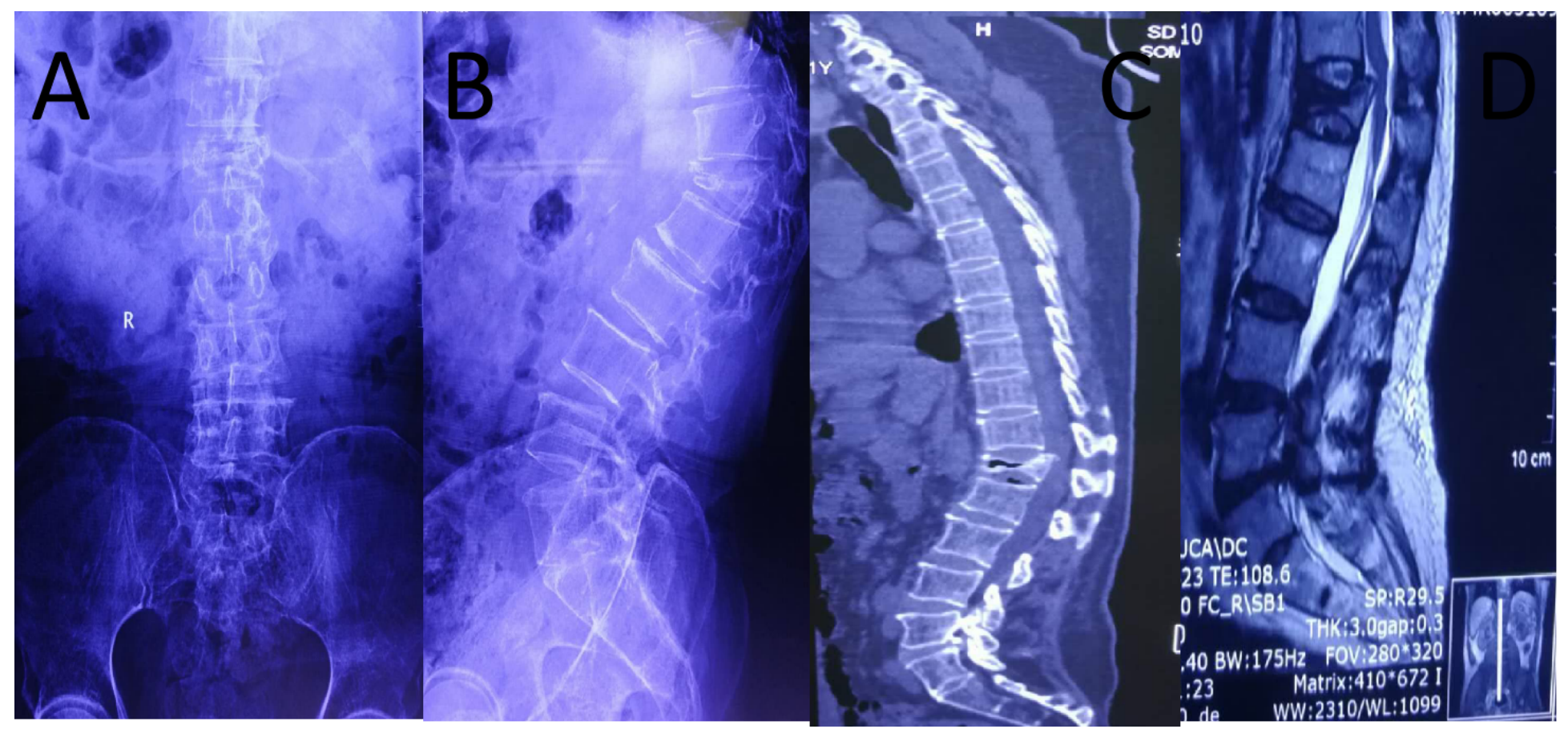

Figure 1

Preoperative images. A and B show the radiograph of positive and lateral position of the spine; $C$ and $D$. CT scans and MRI study of the spine, respectively. Collapse of the T12 vertebral body and "intravertebral vacuum" was shown, suggesting Kümmell disease with kyphosis. 


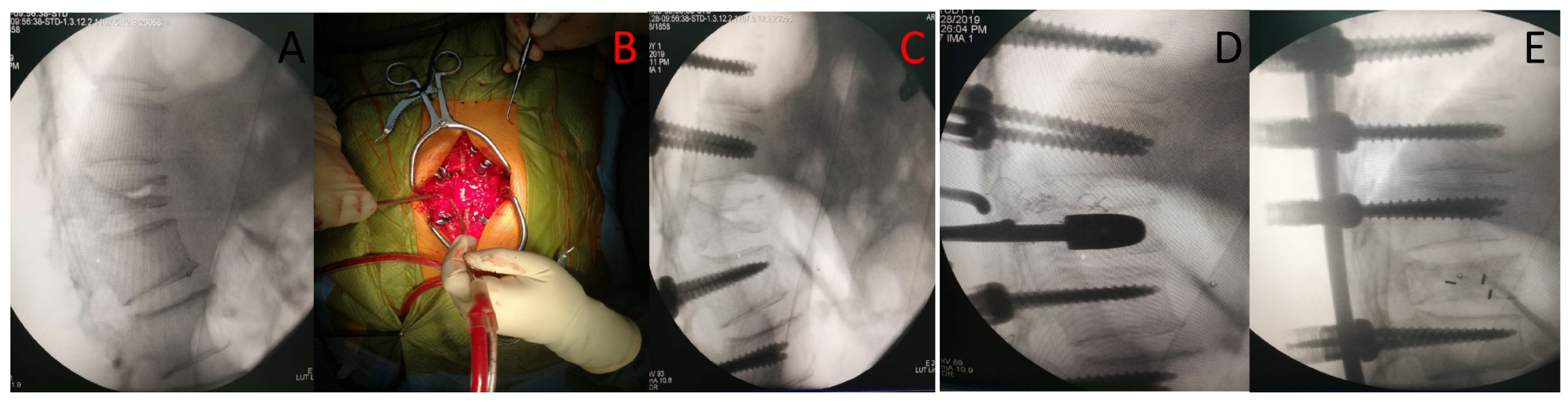

Figure 2

Intraoperative images. Lateral radiograph of the spine under natural distraction(A); Intraoperative photograph (B); image after pedicle screws insertion (C); image of test mold insertion(D); Intraoperative image after bone grafting and appropriate size cage insertion(E). 


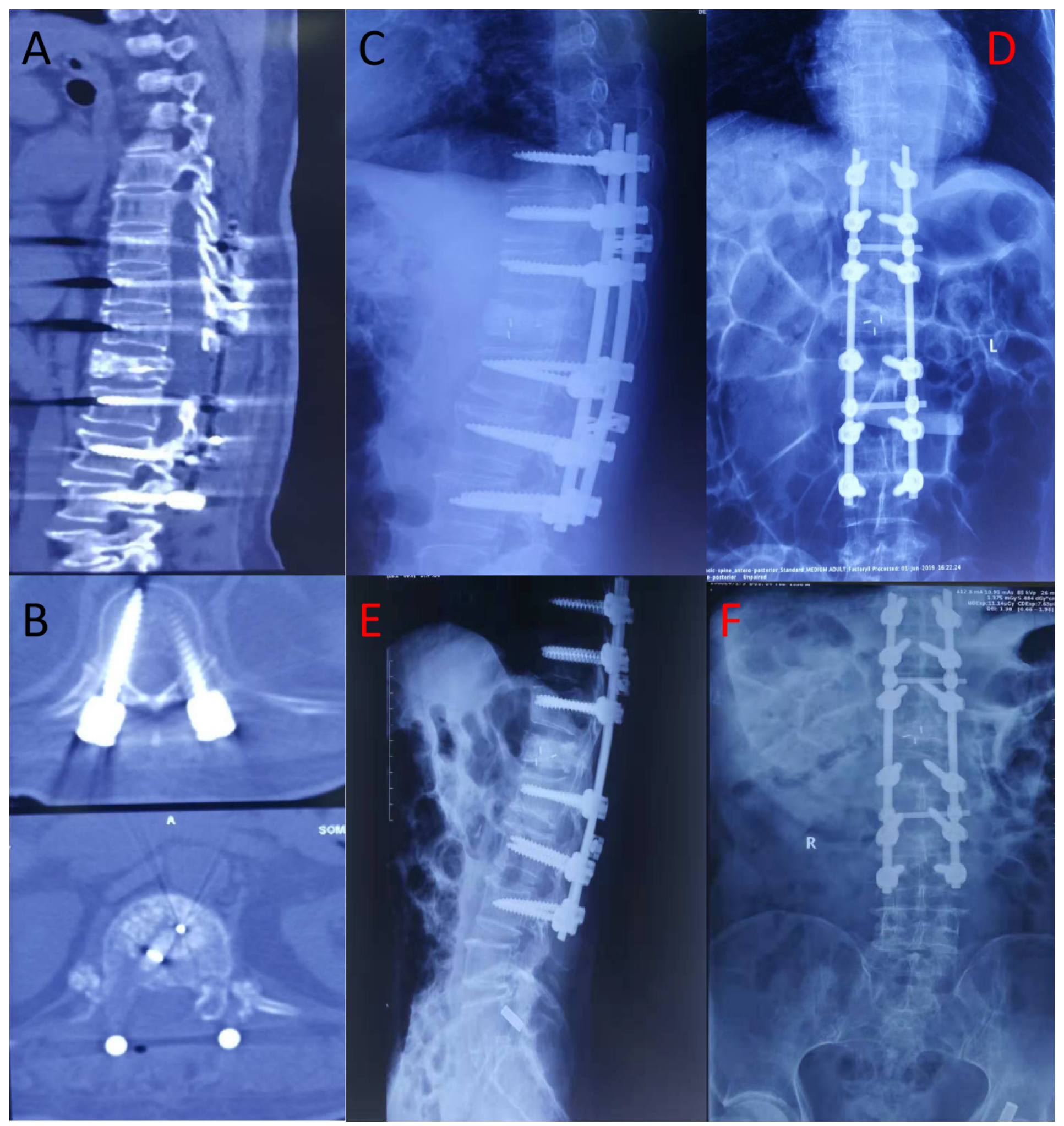

\section{Figure 3}

Images of follow-up postoperatively. A/B shows CT scans on the third-day after surgery, which successfully restored the body height of collapsed T12 vertebral body. C/D shows radiographs at 1 week postoperatively, which demonstrated that the implanted interbody cage in the T12 vertebral body was stable. E/F shows radiographs at 3 months postoperatively, which showed appropriate bone ingrowth in the implanted interbody cage and T12 vertebral body maintained satisfied height. 

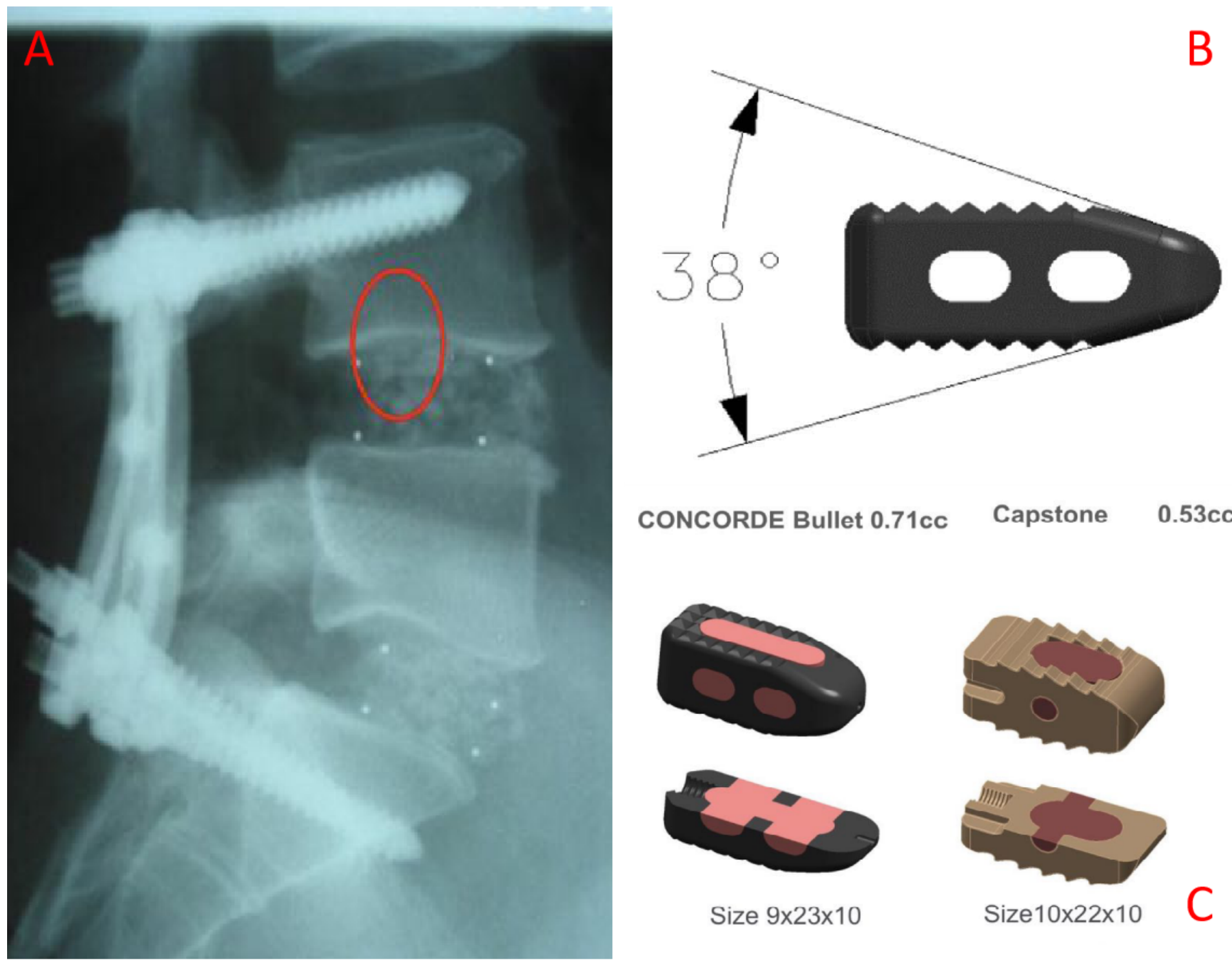

CONCORDE Bullet $0.71 \mathrm{cc}$ Capstone 0.53cc

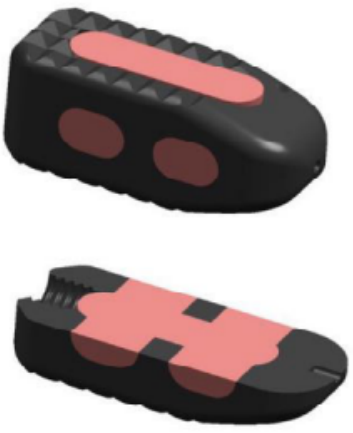

Size $9 \times 23 \times 10$
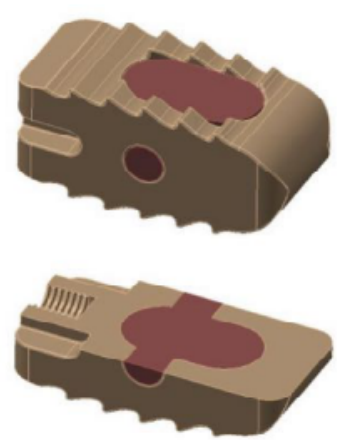

Size10x22x10

\section{Figure 4}

Images of different kinds of interbody cage and its fundamentally application. A shows the initially designed application of interbody cages. B shows the bullethead shape of CFRP fusion cages with a tip angle of 34囚-38囚. C compares the difference between CFRP fusion cage and CAPSTONE cage. 